\title{
A LVRT Control Strategy Based on the Improved Second Order Generalized Integral PLL
}

\author{
Hongling Lei ${ }^{1, *}$,Chang Yang ${ }^{2}$, Jianfei $\mathrm{Li}^{2}$ and Yunxiang $\mathrm{Xie}^{3}$ \\ ${ }^{1}$ School of Electrical Engineering, Guangdong Mechanical \& Electrical College, Guangzhou, China \\ ${ }^{2}$ Sineng Electric Co., Ltd, Shenzhen, China \\ ${ }^{3}$ School of electric power, South China University of Technology, Guangzhou, China \\ ${ }^{*}$ Corresponding author
}

\begin{abstract}
Facing the unbalance drop of three-phase grid voltage, jumping frequency and other complex working conditions, the accuracy and dynamic response of phase-locked loop (PLL) have become the important performance indicators of the photovoltaic grid-connected inverter. Aiming at the problem that the low accuracy of SOGI unit in the traditional double-order generalized integral phase-locked loop (DSOGI-PLL), when the frequency variation of the grid is happened. Thus, the paper proposes a compensation and improved algorithm that the stability of output signal is ensured by the stationary input frequency and the extra compensation. Finally, simulation and experiment show that the proposed PLL system can filter out the harmonic part of the grid voltage, but also, lock the phase fast and accurately. And it ensures that the grid-connect inverter goes through the fault. So that the PLL has strong adaptability to the grid.
\end{abstract}

Keywords-phase-locked loop; second order generalized integrator; low voltage ride through

\section{INTRODUCTION}

In recent years, renewable energy power generation system is booming, power station scale is expanding, and the market gradually is maturing(1). At the same time, a large number of devices and equipment are connected into the grid, which would lead to the low stability of the grid. Especially when the grid is faulty, the phase-locked loop accuracy is particularly important. The phase-locked loop based on the synchronous reference coordinate system (SRF-PLL) is simple and good dynamic performance. However, under the aberration case of grid voltage, it cannot accurately detect the synchronization signal, which affects the stability of the power system(2). The phase-locked loop based on the dual-order generalized integrator (DSOGI-PLL) can detect the voltage synchronization signal accurately, under the asymmetrical and low-order harmonics case the grid fault produces. Now it is widely used in engineering (3) (4). However, the second order generalized integrator (SOGI) unit in the DSOGI-PLL operates in a frequency-adaptive manner. Namely, the input frequency signal is derived from the frequency feedback output from the later stage-locked loop. It makes the phase-locked system more complex. But also, when the grid frequency is changed, the two input signals of the SOGI unit which contain the amount of disturbance are easy to fluctuate the output signal. Which leads to a low performance of the DSOGI-PLL phase lock and a reduction in system stability (5).
The reason for the unbalanced voltage and frequency variation is analyzed. Because of the shortcomings of the output fluctuation of the traditional SOGI unit, an improved method is proposed to ensure the stability of the output signal by fixing the input angular frequency and adding the compensation term. Finally, the reliability of the improved system is verified by low-voltage ride through (LVRT) experiment.

\section{FAUlt StATE ANALysis OF GRID Voltage UNBALANCE}

In three-phase three-wire system, when the three-phase unbalanced voltage drop occurs, the zero sequence component can be ignored. The voltage can be expressed as the sum of positive and negative sequence components:

$$
u_{i}=U_{p} \cos \left(\omega t+\phi_{p}-k_{i} \frac{2 \pi}{3}\right)+U_{n} \cos \left(-\omega t+\phi_{n}-k_{i} \frac{2 \pi}{3}\right)
$$

When $i=a, b, c, k_{i}=0,1,2 . U_{p}, U_{n}, \phi_{p}, \phi_{n}$ are the positive and negative sequence components of the fundamental amplitude and the initial phase angle respectively; $\omega$ is the fundamental frequency of the grid.

In PLL normal operation, the phase angle of the voltage positive sequence component is equal to the rotation angle of the rotation coordinate axis, namely $\theta_{P}=\omega t+\phi_{P}$. And the grid voltage is shown as:

$$
\left[\begin{array}{l}
u_{d} \\
u_{q}
\end{array}\right]=U_{P}\left[\begin{array}{l}
1 \\
0
\end{array}\right]+U_{N}\left[\begin{array}{l}
\cos \left(-2 \omega t+\phi_{N}-\theta_{P}\right) \\
\sin \left(-2 \omega t+\phi_{N}-\theta_{P}\right)
\end{array}\right]
$$

When the grid voltage is asymmetric, through the Clark and Park transformation, the grid voltage baseband positive and negative sequence components were converted to DC and two times the frequency of the AC component in dq axis. The double frequency component makes the SRF-PLL in the grid asymmetrical drop produce double frequency oscillation. 


\section{Phase Locking Scheme ANd Control StRATEgy}

\section{A. Phase Locking Scheme Based on Double Second Order Generalized Integrator}

In the engineering, double second order generalized integrator (DSOGI-PLL) is commonly used. It can suppress the second harmonic under the grid unbalance failure, and has high stability. According to the symmetric component method, the positive sequence voltage is:

$$
v_{a b c}^{+}=\left[\begin{array}{lll}
v_{a}^{+} & v_{b}^{+} & v_{c}^{+}
\end{array}\right]^{T}=\left[T_{+}\right] v_{a b c}
$$

According to Clark and Park transformation, it can be derived that:

$$
\begin{aligned}
v_{\alpha \beta}^{+} & =\left[T_{\alpha \beta}\right] v_{a b c}^{+}=\left[T_{\alpha \beta}\right]\left[T_{+}\right] v_{a b c} \\
& =\left[T_{\alpha \beta}\right]\left[T_{+}\right]\left[T_{\alpha \beta}\right]^{-1} v_{\alpha \beta}=\frac{1}{2}\left[\begin{array}{cc}
1 & -q \\
q & 1
\end{array}\right] v_{\alpha \beta}
\end{aligned}
$$

$\mathrm{q}$ is the phase shift factor of $90^{\circ}$. It can be seen from (4) that the positive sequence component of the original grid voltage can be obtained by phase shifting the grid voltage in the rotating coordinate system. SOGI can produce two-phase orthogonal signal. The structure diagram is shown in Figure.1.

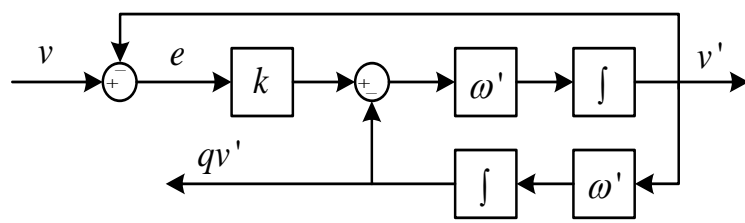

FIGURE I.THE STRUCTURE DIAGRAM OF SOGI

According to Figure.1, SOGI system closed-loop transfer function can be shown as:

$$
\begin{gathered}
D(s)=\frac{v^{\prime}}{v}(s)=\frac{k \omega^{\prime} s}{s^{2}+k \omega^{\prime} s+\omega^{\prime 2}} \\
Q(s)=\frac{q v^{\prime}}{v}(s)=\frac{k \omega^{\prime 2}}{s^{2}+k \omega^{\prime} s+\omega^{\prime 2}}
\end{gathered}
$$

According to equation (5) and (6), amplitude-frequency characteristics and phase-frequency characteristics of the system can be shown as:

$$
v^{\prime}=\operatorname{Dv}\left\{\begin{array}{l}
|D|=\frac{k \omega \omega^{\prime}}{\sqrt{\left(k \omega \omega^{\prime}\right)^{2}+\left(\omega^{2}-\omega^{\prime 2}\right)^{2}}} \\
\angle D=\tan ^{-1}\left(\frac{\omega^{\prime 2}-\omega^{2}}{k \omega \omega^{\prime}}\right)
\end{array}\right.
$$

$$
q v^{\prime}=\mathrm{Q} v\left\{\begin{array}{l}
|Q|=\frac{\omega^{\prime}}{\omega}|D| \\
\angle Q=\angle D-\frac{\pi}{2}
\end{array}\right.
$$

In the steady state, $\omega^{\prime}=\omega$, then D (s) and Q (s) amplitudefrequency characteristics are 1 , namely, $v^{\prime}=v$, and the phasefrequency characteristics differ by $90^{\circ}$.

The specific process of the DSOGI-PLL is shown in Figure. 2. After putting the obtained quadrature signal into the equation (4), the positive voltage of the grid voltage in the coordinate system can be obtained. Then, the positive voltage is transformed into the components in $\mathrm{d}-\mathrm{q}$ axis by Park transformation. There are not the second-order harmonic components in the components in d-q axis. Finally, the output of the DSOGI-PLL is equal to the grid voltage phase angle, through the traditional phase lock mode. Namely, the reactive components are zero.

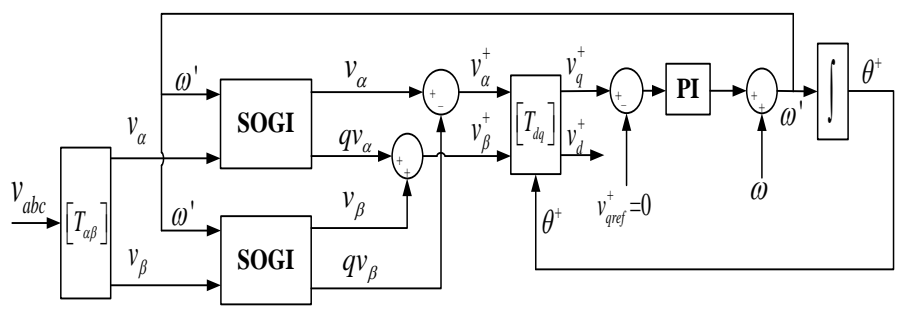

FIGURE II.

THE STRUCTURE DIAGRAM OF DSOGI-PLL

\section{B. Improvement Scheme of Second Order Generalized Integrator}

If the grid voltage frequency is changed, the phase-locked loop output angular frequency $\omega^{\prime}$ would be in the oscillation state subsequently. And then SOGI's two input signals contain the amount of disturbance, which will affect the amplitude and phase of $\mathrm{D}(\mathrm{s})$ and $\mathrm{Q}(\mathrm{s})$, further the PLL phase lock accuracy.

In equation (8), $|Q|=|D|$, when $\omega^{\prime}=\omega$. The power frequency of steady state grid voltage is $f_{0}=\omega_{0} / 2 \pi=50 \mathrm{~Hz}$. In this case, the output angular frequency $\omega^{\prime}$ of the PLL can be determined by the voltage frequency $\omega_{0}$ of the power grid and an item $\omega^{\prime} / \omega_{0}$, which is compensated at the $q v^{\prime}$-signal output side. The specific improvements is shown as Figure. 3:

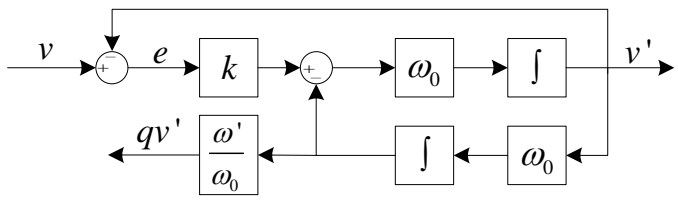

FIGURE III. THE STRUCTURE DIAGRAM OF IMPROVED

After being improved, the Bode diagram of the SOGI system is shown as Figure 4: 

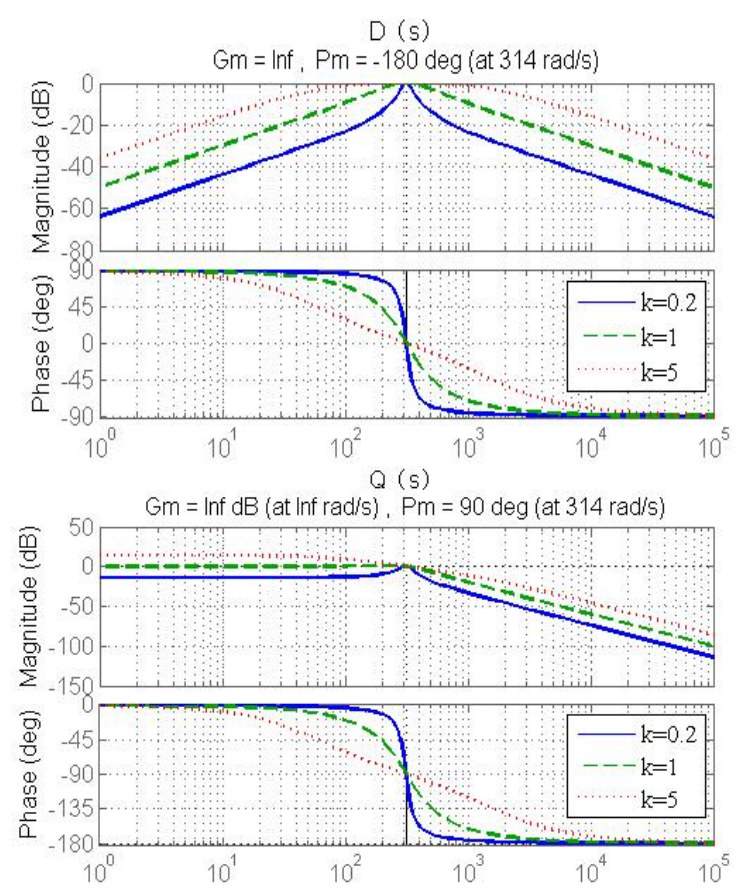

FIGURE IV. THE BODE DIAGRAM OF IMPROVED SOGI

In the Figure.4, it can be seen that $\mathrm{D}(\mathrm{s})$ is a band-pass filter and $\mathrm{Q}(\mathrm{s})$ is a low-pass filter. When $k>1$, the amplitudefrequency characteristic of $\mathrm{Q}(\mathrm{s})$ at $f<50 \mathrm{~Hz}$ is greater than 1 , indicating that the system has an amplification for lowfrequency noise. The smaller the $k$ value, the better the filter frequency resolution, however the slower the system response. Comparing with $k=\sqrt{2}$ in traditional SOGI, $k$ is equal to 0.9 in the improved SOGI.

When the grid is stability, in the Bode diagram, it can be seen that the amplitude attenuations of $\mathrm{D}(\mathrm{s})$ and $\mathrm{Q}(\mathrm{s})$ are 0 , and the phase angle of $\mathrm{Q}(\mathrm{s})$ lags behind $\mathrm{D}(\mathrm{s})$ by $90^{\circ}$ at the frequency of $50 \mathrm{~Hz}$.

When the frequency of the grid is disturbed, because of the compensation $\omega^{\prime} / \omega_{0}$ in the output side of $q v^{\prime}$ signal, the amplitude and phase characteristics of Q(s) are:

$$
\begin{aligned}
& |Q|=\frac{k \omega_{0}^{2}}{\sqrt{\left(k \omega_{0} \omega^{\prime}\right)^{2}+\left(\omega_{0}^{2}-\omega^{\prime 2}\right)^{2}}} \cdot \frac{\omega^{\prime}}{\omega_{0}} \\
& \angle Q=\tan ^{-1}\left(-\frac{\omega_{0}^{2}-\omega^{\prime 2}}{k \omega_{0} \omega^{\prime}}\right)
\end{aligned}
$$

The amplitude and phase characteristics of $\mathrm{D}(\mathrm{s})$ are similar to those of Eq. (7). At this time, D(s) and Q(s) are still orthogonal. However, because of only one perturbation in the amplitude characteristics of $\mathrm{D}(\mathrm{s})$ and $\mathrm{Q}(\mathrm{s})$, the robustness and the anti-interference performance of the system will be greatly enhanced.

\section{Low Voltage Crossing Control Strategy}

According to the LVRT specification, the PV power station needs to have certain LVRT capability in the event of transient fault of the power grid. But also the inverter can submit the reactive power to the grid to support the grid voltage during the fault (5).

In order to solve the problem of asymmetric component caused by unbalanced voltage drop in the grid, double current loop control based on the positive and negative sequences, proposed in the literature (6)(7)(8), used the symmetrical d-q rotation coordinate system to control the positive and negative sequence current. However, in this control mode, four PI controllers are required. And the complex control structure reduces the response speed of the system. This paper chooses to control only the positive sequence current and the negative sequence current is controlled by the negative sequence voltage feed forward. To control the positive sequence current, for example, the specific method is shown in Figure.5.

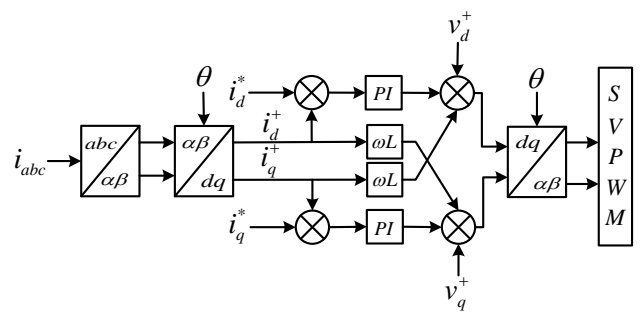

FIGURE V

THE SCHEMATIC DIAGRAM OF CURRENT LOOP IN LVRT STRATEGY

The positive sequence voltage and phase-locked angle $\theta$ of the $\mathrm{d}-\mathrm{q}$ axis are given by the DSOGI-PLL. The deviation between positive sequence current and the reference value goes through the PI controller, then coupled with the decoupling items of the feed forward current. Through the anti-Park transformation and the SVPWM, the signals is transformed into switch drive signals to control the inverter.

In this paper, TMS320F28335 chip is used to control the system. CPU constantly collects three-phase voltage data to determine the case of the grid voltage. When the grid is running normally, the inverter operates in parallel with the power factor 1 . When the voltage is dropped, the fault type and the depth of the drop are judged and the reactive power is used to support the grid voltage.

\section{SimUlation AND EXPERIMENTAL RESUlTS}

In order to verify the correctness of the theoretical analysis, the inverter model and the control loop are set up in the Matlab platform to verify the performance of the DSGI-PLL. The specific control parameters are shown as table 1. PLL $k_{p}$ $=30, k_{i}=0.02$; current control loop $k_{p}=5, k_{i}=200$. The simulation results are shown as Figure.6. 


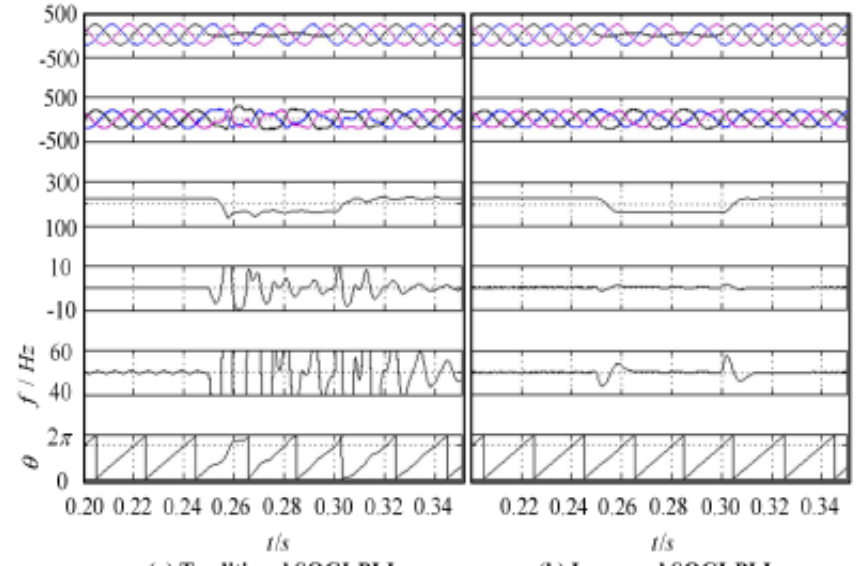

(a) Traditional SOGI-PLL

(b) Improved SOGI-PLL

FIGURE VI. SIMULATION WAVEFORMS

According to the simulation results, it can be seen that the improved DSOGI-PLL can still lock the voltage phase when the voltage drops, and the anti-jamming performance is stronger.

Finally, a $50 \mathrm{~kW}$ three-phase grid-connected inverter experiment platform was built, and the experiment was carried out by the low-voltage ride through control strategy. As the line voltage can only be collected in the three-phase three-wire system, the line voltage corresponds to the phase voltage with $30^{\circ}$ phase difference. Setting the grid voltage RMS 500V, the experimental waveforms shown in Figure.7.

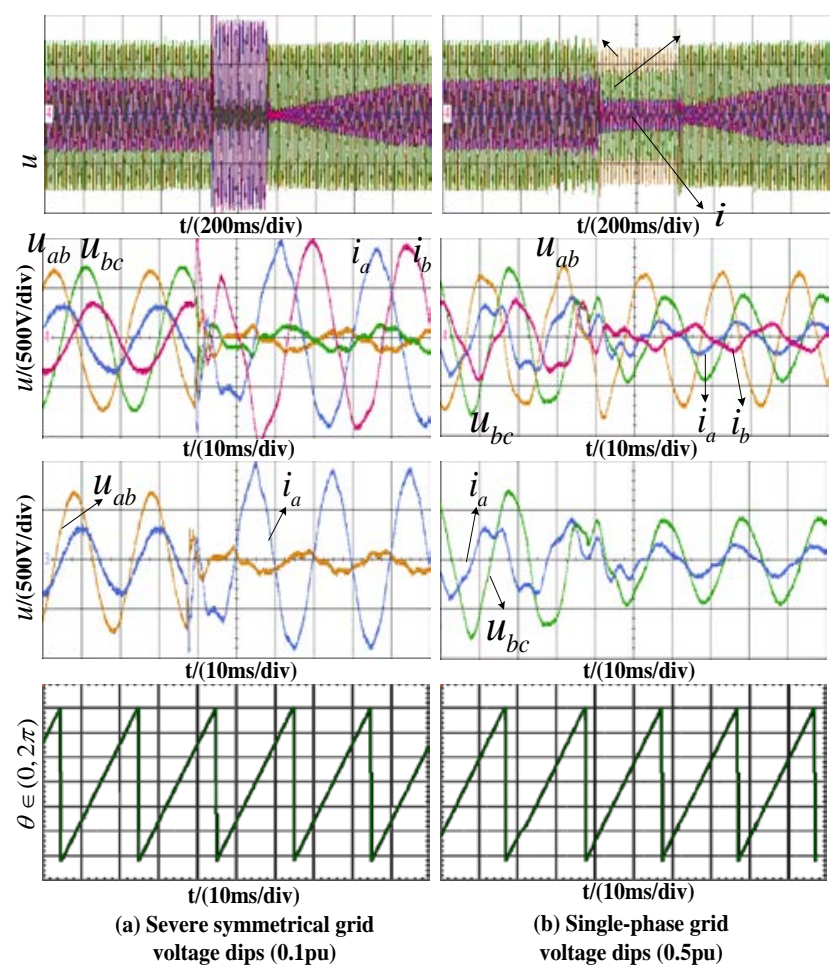

FIGURE VII.

EXPERIMENTAL WAVEFORMS

According to the experimental results, it can be seen that the improved DSOGI-PLL can successfully locked the phase during low-voltage ride through. The changed phase of current before and after the voltage drop, indicates that the system generates reactive power to support the grid voltage after the voltage is reduced.

\section{IN CONCLUSION}

In this paper, the phase-locked state of PV grid-connected inverter in the events of grid voltage asymmetry is studied. Aiming at the problem that the DSOGI-PLL using the traditional SOGI unit is unstable when the asymmetric component is generated during the power grid fault, an improved strategy is proposed. In the strategy, fixed-angle frequency and the compensation ensure the signal stability. The simulation results show that the DSOGI-PLL can improve the phase of the grid accurately, and has a simpler structure and a stronger power grid adaptability. During low-voltage ride through, the system can quickly control the inverter to generate reactive power to support the grid voltage.

\section{REFERENCES}

[1] Xiaodong Yang, Yu Chen, Youbing Zhang, et al. Scheduling optimization model for microgrid considering interactive power and renewable energy output fluctuation[J]. Power System Protection and Control, vol.44, no. 23, pp. 30-38 ,Dec. 2016.

[2] Xianbin Huang, Da Lin, Huifang Wang, and Tao Wu, "Summary of low voltage crossing strategy for grid - connected PV system," Electrical and Mechanical Engineering, vol.33, no. 5, pp. 589-594 ,Aug. 2016.

[3] Shukui Liu, Ying Han, and Qi Li , "Research on grid-connected system of fuel cell based on bi-second-order generalized phase-locked loop,” Power System Protection and Control, vol.42, no. 5, pp. 122-128, May 2014.

[4] Cong Xie, Xinkai Wu, and Yayun Lei, "Wind power grid-connected system simulation based on double-second generalized integral phaselocked,” Electric Drive Automation, vol.37, no. 2, pp. 1-5, May 2015.

[5] Laiying Xi , Jinfu Wang , Chuanchuan Hou,et al. Design of phase locked loop based on third-order general-integrator[J]. Power System Protection and Control, vol.44, no. 23, pp. 184-189 ,Dec. 2016.

[6] Jianwen Li, Yaxiong Lei, and Yonggang Li, "Grid-connected inverter voltage reference current value ,'Transactions of China Electrotechnical Society, vol.30, no.10, pp. 276-282, Jul. 2015.

[7] Xiaolan Wang, Zhenhua Dong, and Chen Liang, "Voltage stabilization control strategy of photovoltaic power generation system under asymmetric fault ,’Electric drive, vol.44, no.1 2, pp.44-48, Jan.2015.

[8] Lijun Jin, and Yifan Cheng. "Unbalanced control of grid-side converter based on DSOGI-PLL,"2015 IEEE 10th Conference on Industrial Electronics and Applications (ICIEA), pp.1145-1149, 2015. 